\title{
On Numerical Simulation of Electromagnetic Field Effects in the Combustion Process
}

\section{Harijs Kalis ${ }^{a}$, Maksims Marinaki ${ }^{a}$, Uldis Strautins ${ }^{a}$ and Maija Zake ${ }^{b}$}

\author{
${ }^{a}$ Institute of Mathematics and Computer Science of University of Latvia \\ Raina bulvāris 29, LV-1459 Rīga, Latvija \\ ${ }^{b}$ Institute of Physics, University of Latvia \\ 32 Miera Street, LV-2169 Salaspils-1, Latvia \\ E-mail(corresp.): kalis@lanet.lv \\ E-mail: maksims.marinaki@lu.lv \\ E-mail: uldis.strautins @lu.lv \\ E-mail: mzfi@sal.lv
}

Received July 22, 2017; revised March 23, 2018; accepted March 25, 2018

\begin{abstract}
This paper deals with a simplified model taking into account the interplay of compressible, laminar, axisymmetric flow and the electrodynamical effects due to Lorentz force's action on the combustion process in a cylindrical pipe. The combustion process with Arrhenius kinetics is modelled by a single step exothermic chemical reaction of fuel and oxidant. We analyze non-stationary PDEs with 6 unknown functions: the 3 components of velocity, density, concentration of fuel and temperature. For pressure the ideal gas law is used. For the inviscid flow approximation ADI method is used. Some numerical results are presented.
\end{abstract}

Keywords: compressible, laminar, axisymmetric flow, Lorentz force, Arrhenius kinetics.

AMS Subject Classification: 65N06; 65N20; 65N40; 65N25; 34C55; 65N35.

\section{Introduction}

The use of swirling flows to control the combustion processes is actual and essential, because of the hypothesis suggesting that the swirl flows allow the enhanced mixing of the reactants in the flame reaction zone and stabilization of the processes of fuel combustion and heat energy production. Within the last years, biomass combustion for energy purposes has gained rising popularity. The studies of Syred, Gupta and Lilley [14], [23], [20] provide analysis of the

Copyright (c) 2018 The Author(s). Published by VGTU Press

This is an Open Access article distributed under the terms of the Creative Commons Attribution License (http://creativecommons.org/licenses/by/4.0/), which permits unrestricted use, distribution, and reproduction in any medium, provided the original author and source are credited. 
effect of the swirl level (S) on the swirl flow dynamics and structure formation of the flame reaction zone. Barmina et al. [5] the experimental study of the formation of the flame composition and temperature profiles downstream the swirling flame flows are carried out. The complex research of the swirl flow formation has shown that the formation of the compact recirculation zone is observed at $S>0.6$.

Ahmed and Das [3] have obtained that the chemical reaction and the heat sink have significant effects on the flow and on the heat and mass transfer characteristics. Battaglija et al. [9] has described the mathematical models of the hydrodynamics and the combustion; with the large-eddy simulation (LES) he has presented the temperature fields, swirl velocity characteristics and the heat release rate. Bayona and Kindelan [10] have simulated the premixed laminar flames with different values of Lewis number in the open ducts with the spectral finite difference metd. Mittal et al. [22] has used the laminar burning velocity has used for modelling the premixed combustion process. The quasi -gasdynamic system of equations with the mass force and heat source for the perfect polytropic gas has been studied in [26]. In [25] Zlotnik and Čiegis have investigated the stability for the high order finite difference scheme.

In the paper of Choi, Rusak et al. [12] a numerical investigation of the inviscid, axisymmetric, steady swirling flow in cylindrical pipe for low Mach number approximation is developed $\left(\rho=\frac{1}{T}\right.$, where $\rho, T$ are the dimensionless density and the temperature in compressible flow). For numerical simulation the pressure-correction method is used.

The investigation continues the study of Kalis et al. [4], [7], [17], [18], where the pressure is eliminate from the Navier-Stokes equations for viscous, incompressible and ideal, compressible flow by introducing the stream function and vorticity for the combustion process with simple exothermic chemical reaction. The swirling flow with axial and azimuthal components of velocity is developed in the pipe. The axial velocity from uniform stream in the central part of the cylindrical pipe-inlet is formed. The azimuthal velocity with rotation of the part of tube inlet is obtained.

In [18] the results of numerical study of viscous, incompressible, laminar, axisymmetric swirling flow with axial uniform magnetic field are presented. The interplay of two strategies - inducing swirl in the flow combined the application of an external magnetic field is considered. The swirl number is introduced by controlling the axial and azimuthal velocity components at the inlet. A similar experiment is reported in [4]. The external axial magnetic field is modeled for the Lorentz force. The flow field is influenced by gravitation, magnetic field, heat of reaction and swirl number. Since the conductivity of hot gases such as air, oxygen, hydrogen is very low, the MHD mechanisms are not very effective in practical applications of gas combustion. This is in contract with conductive liquid, for which external magnetic field can be effectively used to control the vorticity distribution in the flow [16].

In [17] inviscid, compressible, laminar, axisymmetric steady swirling flow is numerically investigated. From the results one can conclude that the increase in value of axial velocity in terms of maximal temperature of reaction $T_{\max }$ leads to increase for $L e>1$ and decrease for $L e<1$, where $L e=\frac{\lambda}{c_{p} D}$ is Lewis 
number $\left(\lambda\right.$ is the thermal conductivity, $c_{p}$-the specific heat, $D$-the molecular diffusivity). Similar results are obtained in [2] for second-order reaction kinetics for fast and slow exothermic reactions in the combustion of $\mathrm{CO}$ and $\mathrm{H}_{2}$.

In [4] the electromagnetic force is generated by an electric current in a coil electrode surrounding the flame at the inlet of the cylindrical pipe and the azimuthal component of vector potential and Biot-Savart law were used to investigate and calculate the distribution of the electromagnetic field axial and radial components. It has been proved that the increase of electrodynamic force increases the maximal velocity in the gas flow.

In [7] the magnetic field is induced by direct electric current in a coil, which is placed at inlet of the combustor, close to the inner surface of the combustor. The distribution of stream function, azimuthal component of velocity, vorticity and formation of temperature profiles are calculated by varying the electrodynamic force and swirl number.

In $[6,8,15]$ a 2D axially-symmetric ideal, compressible swirling flow with simple chemical raction is descibed by four Euler and two reaction-diffusion equations. The perfect gas model $p=\rho T$ has been used ( $p$ is the dimensionless pressure). The approximation is based on implicit finite-difference and alternating direction(ADI) method of Douglas and Rachford [13]. The applied electric field induces an electrical current between the positively based walls of the combustor and the negative biased axially inserted electrode with different length. The field influence on the flame length depends on the length of the axially inserted electrode. The vortex breakdown at swirl number $S>0.9$ in the recirculation zone is obtained.

In [1] a nonlinear thermal conductivity model in two gypsum product layers with different density and high temperature $\left(T>500^{\circ} \mathrm{C}\right)$ is proposed. The conservative averaging method (CAM) allows reducing the nonlinear 2-D heat transfer initial-boundary problem to the initial value problem for system of ODEs of first order.

In this paper we focus on a configuration in which a steady, low-speed $\left(0.1 \frac{\mathrm{m}}{\mathrm{s}}\right)$, laminar flame exists in a straight pipe in the base state (in previous investigation the axial velocity has a uniform stream $\left.U_{0}=0.01\left[\frac{\mathrm{m}}{\mathrm{s}}\right]\right)$. We consider a simplified model taking into account the interplay of compressible, laminar, axisymmetric flow and the electrodynamical effects due to Lorentz force's action on the combustion process in a cylindrical pipe.

Similarly to [15] the axial velocity from uniform stream in the central part of the cylindrical pipe-inlet is formed, the azymuthal velocity with rotation of the part from tube inlet is obtained. The fuel (propane) is injected axially into the sectioned water-cooled channel, air swirl motion is generated through a tangential air inlet. Therefore, the air swirl flow combines axial and azimuthal motions. The exothermic chemical reaction is modelled by a single step of fuel and oxidant. The rate of the reaction is given by the one-step first-order Arrhenius kinetics. Our purpose is to understand how the stationary flame is affected by the introduction of swirl and direct electric current, whish is fed to discrete circular conductor-electrode or by an external magnetic field which is induced by a permanent magnetic material wrapped around the cylinder.

The distribution of axial, radial and azimythal components of velocity, vor- 
ticity, density, and temperature has been calculated using the implicit finite difference method and ADI method. For 1D reaction-diffusion problem some results are obtained with Matlab solvers "pdepe" and "bvp4c".

\section{The mathematical model}

The combustion process with temperature $T[K]$ and simple exothermic chemical reaction is modelled by the first-order Arrhenius kinetics with mass fraction $C$ of the reactant in time $t$, in the coaxial cylindrical pipe with radius $r_{0}=0.05[\mathrm{~m}]$ and length $z_{0}=0.1[\mathrm{~m}]$. The axial, radial and azimuthal components of the velocity $u_{z}, u_{r}, u_{\theta}$ in the coaxial cylindrical pipe are formed.

Let $T_{0}=300[K], \rho_{0}=1\left[\frac{\mathrm{kg}}{\mathrm{m}^{3}}\right], C_{0}=1$ be the initial temperature, nominal density, mass fraction of concentration of fuel and axial velocity with uniform stream $U_{0}=0.1\left[\frac{\mathrm{m}}{\mathrm{s}}\right]$ in the central part of the cylindrical pipe at inlet $z=0$.

The boundary of the pipe $\left(r=r_{0}\right)$ is subject to a heat loss modelled by the Newtonian cooling to the ambient surroundings at temperature $T_{0}$ and with heat transfer coefficient $h=0.1\left[\frac{J}{s m^{2} K}\right]$.

Direct electric current with the meridian components of the vector density $j=\left(j_{z}, j_{r}, 0\right)\left[\frac{A}{m^{2}}\right]$ and current $I[A]$ is fed to axially-symmetric conductorelectrodes: $L_{1}=\left\{\left(z, r_{0}, \phi\right), 0 \leq z \leq z_{1}<z_{0}, 0 \leq \phi \leq 2 \pi\right\}$ (the part of walls of the pipe) and $L_{2}=\left\{\left(z, r_{*}, \phi\right), 0 \leq z \leq z_{2}<z_{0}, 0 \leq \phi \leq 2 \pi\right\}$ (the central part of the bottom of the pipe $\left.r_{*}=r_{0} / 10\right)$.

From the Maxwell's equations and Ohm's law it follows [11], that the meridian components of the vector density $j_{z}, j_{r}$ create the azimuthal component $B_{\phi}$ of the induced magnetic field $\left(j_{z}=\frac{1}{\mu r} \frac{\partial\left(r B_{\phi}\right)}{\partial r}, j_{r}=-\frac{\partial B_{\phi}}{\mu \partial z}\right)$ in the ionized gas, which creates the axial $F_{z}=B_{\phi} j_{r}$ and radial $F_{r}=-B_{\phi} j_{z}$ components of the electromagnetic force, where $\mu$ is the magnetic permeability in medium.

We analyze the 2D axially-symmetric nonstationary physical model for the inviscid, compressible, swirling flow with the meridian components of the vector velocity $u_{r}, u_{z}$, circulation $v=r u_{\phi}$, simple chemical reaction and electromagnetic force in cylindrical pipe, which can be described with 4 Euler's and 2 reaction-diffusion equations:

$$
\begin{aligned}
& \frac{\partial \rho}{\partial t}+u_{r} \frac{\partial \rho}{\partial r}+u_{z} \frac{\partial \rho}{\partial z}+\frac{\rho}{r} \frac{\partial\left(r u_{r}\right)}{\partial r}+\rho \frac{\partial u_{z}}{\partial z}=0, \\
& \frac{\partial u_{r}}{\partial t}+u_{r} \frac{\partial u_{r}}{\partial r}+u_{z} \frac{\partial u_{r}}{\partial z}-\frac{v^{2}}{r^{3}}=-\frac{\partial p}{\rho \partial r}+F_{r} / \rho, \\
& \frac{\partial u_{z}}{\partial t}+u_{r} \frac{\partial u_{z}}{\partial r}+u_{z} \frac{\partial u_{z}}{\partial z}=-\frac{\partial p}{\rho \partial z}+F_{z} / \rho, \\
& \frac{\partial v}{\partial t}+u_{r} \frac{\partial v}{\partial r}+u_{z} \frac{\partial v}{\partial z}=0, \\
& \frac{\partial T}{\partial t}+u_{r} \frac{\partial T}{\partial r}+u_{z} \frac{\partial T}{\partial z}=\frac{\lambda}{\rho c_{p}} \nabla^{2} T+\frac{\tilde{B}}{c_{p}} A C \exp \left(-\frac{E}{R T}\right), \\
& \frac{\partial C}{\partial t}+u_{r} \frac{\partial C}{\partial r}+u_{z} \frac{\partial C}{\partial z}=D \nabla^{2} C-A C \exp \left(-\frac{E}{R T}\right),
\end{aligned}
$$


where $\nabla^{2} q=\frac{\partial^{2} q}{\partial z^{2}}+\frac{1}{r} \frac{\partial}{\partial r}\left(r \frac{\partial q}{\partial r}\right), q=T, C, D=5.10^{-5}\left[\frac{m^{2}}{s}\right]$ is the molecular diffusivity, $\lambda=5.10^{-5}\left[\frac{\mathrm{J}}{\mathrm{s}, \mathrm{mK}}\right]$ is the thermal conductivity, $c_{p}=1000\left[\frac{\mathrm{J}}{\mathrm{kgK}}\right]$ is the specific head at constant pressure, $\tilde{B}=1.510^{6}\left[\frac{\mathrm{J}}{\mathrm{kg}}\right], A=10^{4}\left[\frac{1}{\mathrm{~s}}\right], E=$ $2.510^{4}\left[\frac{\mathrm{J}}{\mathrm{mol}}\right]$ are the specific heat release, the reaction-rate pre-exponential factor and the activation energy, $R$ is the universal gas constant. The azimuthal component of the induced magnetic field can be obtained from the following equation of conjugate Laplace operator

$$
\frac{\partial^{2} B_{\phi}}{\partial z^{2}}+r \frac{\partial}{\partial r}\left(\frac{1}{r} \frac{\partial B_{\phi}}{\partial r}\right)=0
$$

For the permanent magnet has a different form of the radial and axial components of the Lorentz force. The FEMM software provides a finite element solution of the Maxwell equations formulated for the magnetostatic case in two spatial dimensions [21]:

$$
\nabla \times \boldsymbol{H}=0, \nabla \cdot \boldsymbol{B}=0, \quad \boldsymbol{B}=\mu(\boldsymbol{H}+\boldsymbol{M}),
$$

where $\boldsymbol{H}$ is the magnetic field intensity, $\boldsymbol{B}$ is the magnetic flux density, $\boldsymbol{M}$ stands for magnetization.

The software considers the vector potential formulation in the case of two dimensions. Zero vector potential boundary condition was applied to the symmetry part of the boundary for the case of axial symmetry. For magnetic flux density, two magnetization directions, perpendicular to the flow direction and aligned with the flow direction, were considered (see Figure 1). The flux density varies between 0.001 Tesla and 0.1 Tesla. For the calculations, a the mean value of 0.03 Tesla has been considered.

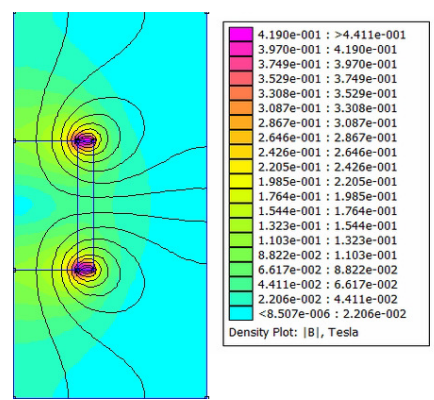

(a) the magnetization direction perpendicular to the flow direction

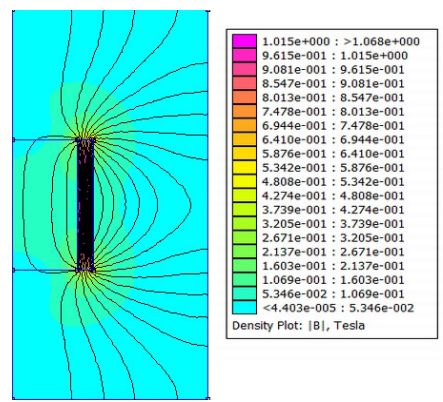

(b) the magnetization direction aligned with the flow direction

Figure 1. Magnetic flux density plot.

After the field $\boldsymbol{B}$ with the two components $B_{r}, B_{z}$ has been obtained, we use the Matlab built-in differentiation commands to calculate the Lorentz force terms in the following form:

$$
F_{r}=(\nabla \times \boldsymbol{B})_{\phi} B_{z}, \quad F_{z}=(\nabla \times \boldsymbol{B})_{\phi} B_{r}, \quad(\nabla \times \boldsymbol{B})_{\phi}=\frac{\partial B_{r}}{\partial z}-\frac{\partial B_{z}}{\partial r} .
$$


For the pressure we use the ideal gas law : $p_{0}=R T_{0} \rho_{0} / M^{\prime}\left[\frac{N}{m^{2}}\right]$, where $M^{\prime}=$ $0.0032\left[\frac{\mathrm{kg}}{\mathrm{mol}}\right]$ is the molar mass for $\mathrm{O}_{2}[24]$.

The equations were put in the dimensionless form scaling all the lengths to $r_{0}$, the density to $\rho_{0}$, the velocities $u_{r}, u_{z}$ to $U_{0}$, the circulation $v$ to $V_{0} r_{0}$ $\left(V_{0}>U_{0}\right.$ is the tangential air component), the pressure $p$ to $p_{0}=\rho_{0} U_{0}^{2}$, the temperature to $T_{0}$, the magnetic induction $B_{\phi}$ to $B_{0}=\frac{\mu I}{2 \pi r_{0}}[$ Tesla], Tesla $=$ $\frac{N}{A . m}$, the electric current densities $j_{r}, j_{z}$ to $j_{0}=\frac{I}{2 \pi r_{0}^{2}}\left[\frac{A}{m^{2}}\right]$, the electromagnetic forces $F_{r}, F_{z}$ to $F_{0}=j_{0} B_{0}\left[\frac{N}{m^{3}}\right]$, the special heat release $\tilde{B}$ to $\frac{c_{p}}{T_{0}}$, the reactionrate pre-exponential factor $A$ to $\frac{U_{0}}{r_{0}}$, the activation energy $E$ to $\frac{R}{T_{0}}$.

The following parameters are used: $P e=\frac{r_{0} U_{0}}{D}, L e=\frac{\lambda}{c_{p} D \rho_{0}}$ are Peclet and Lewis mumbers, $P_{1}=\frac{L e}{P e}, P_{2}=\frac{1}{P e}, S=\frac{V_{0}}{U_{0}}$ is the swirl number, $P_{e}=\frac{F_{0} r_{0}}{\rho_{0} U_{0}^{2}}$ is the electrodynamical force parameter, $\beta=\frac{\tilde{B}}{c_{p} T_{0}}, \delta=\frac{E}{R T_{0}}$ are the scaled heat release and the activation energy.

For the dimensionless parameters $t, r, x=z / r_{0}, \rho, u=u_{r} / U_{0}, w=u_{z} / U_{0}$, $v$ we have the following equations

$$
\begin{aligned}
& \frac{\partial \rho}{\partial t}+u \frac{\partial \rho}{\partial r}+w \frac{\partial \rho}{\partial x}+\frac{\rho}{r} \frac{\partial(r u)}{\partial r}+\rho \frac{\partial w}{\partial x}=0, \\
& \frac{\partial u}{\partial t}+u \frac{\partial u}{\partial r}+w \frac{\partial u}{\partial x}=S^{2} \frac{v^{2}}{r^{3}}-\frac{\partial p}{\rho \partial r}+P_{e} F_{r}^{\prime} / \rho, \\
& \frac{\partial w}{\partial t}+u \frac{\partial w}{\partial r}+w \frac{\partial w}{\partial x}=-\frac{\partial p}{\rho \partial x}+P_{e} F_{x}^{\prime} / \rho, \\
& \frac{\partial v}{\partial t}+u \frac{\partial v}{\partial r}+w \frac{\partial v}{\partial x}=0, \\
& \frac{\partial T}{\partial t}+u \frac{\partial T}{\partial r}+w \frac{\partial T}{\partial x}=\frac{P_{1}}{\rho} \nabla^{2} T+\beta A C \exp \left(-\frac{\delta}{T}\right), \\
& \frac{\partial C}{\partial t}+u \frac{\partial C}{\partial r}+w \frac{\partial C}{\partial x}=P_{2} \nabla^{2} C-A C \exp \left(-\frac{\delta}{T}\right),
\end{aligned}
$$

where $p=\rho T, F_{r}^{\prime}, F_{x}^{\prime}$ are the dimensionless forces. We introduce the stream function $\Psi$ and the vorticity $\zeta$ with the following expressions:

$$
r \rho w=\frac{\partial \Psi}{\partial r}, \quad r \rho u=-\frac{\partial \Psi}{\partial x}, \quad \zeta=\frac{\partial u}{\partial x}-\frac{\partial w}{\partial r} .
$$

Then we have the following equation for the stream function $\Psi$ :

$$
\frac{\partial \Psi}{\partial t}=\frac{\partial}{\partial x}\left(\rho^{-1} \frac{\partial \Psi}{\partial x}\right)+r \frac{\partial}{\partial r}\left(\frac{1}{\rho r} \frac{\partial \Psi}{\partial r}\right)+r \zeta
$$

where the equation for the numerical simulation is transformed to non-steady. The approach seeks the steady solution as the limit of solutions of the unsteady equations.

The boundary conditions for $B_{\phi}$ on the electrode are the following:

1) On $L_{1}$ from total current condition

$$
I=\int_{0}^{2 \pi} r_{0} \int_{0}^{z_{1}} j_{r}\left(z, r_{0}\right) d z d \phi=-\frac{2 \pi r_{0}}{\mu}\left(B_{\phi}\left(r_{0}, z_{1}\right)-B_{\phi}\left(r_{0}, 0\right)\right)
$$


and from $B_{\phi}\left(r_{0}, z\right)=0, z \in\left[z_{1}, z_{0}\right]$ it follows that $B_{\phi}\left(r_{0}, 0\right)=B_{0}$. From the uniform distribution of current density $j_{r}=$ const on the electrode we get that $B_{\phi}\left(r_{0}, z\right)=-\mu j_{r} z+B_{\phi}\left(r_{0}, 0\right)$ and $B_{\phi}\left(r_{0}, z\right)=B_{0}\left(1-\frac{z}{z_{1}}\right), z \in\left[0, z_{1}\right]$.

2) Similarly on $L_{2}$ we get: $B_{\phi}\left(r_{*}, z\right)=0, z \in\left[z_{2}, z_{0}\right], B_{\phi}\left(r_{*}, z\right)=B_{0}(1-$ $\left.z / z_{2}\right) r_{0} / r_{*}, z \in\left[0, z_{2}\right]$.

On the inlet $z=0$ we have $j_{z}=0$ and $B_{\phi}(r, 0)=B_{0} r_{0} / r$. The other dimensionless BCs are the following:

1) along the wall $r=r^{*}=\frac{r_{*}}{r_{0}}-u=v=0, B_{\phi}\left(r^{*}, x\right)=\left(1-\frac{x}{x_{2}}\right) \frac{1}{r^{*}}, x \in\left[0, x_{2}\right]$,

$$
\frac{\partial T}{\partial r}=\frac{\partial C}{\partial r}=\frac{\partial \rho}{\partial r}=\frac{\partial w}{\partial r}=0, \quad \Psi=0
$$

2) at the wall $r=1-u=v=0, B_{\phi}(1, z)=B_{0}\left(1-x / x_{1}\right), x \in\left[0, x_{1}\right]$,

$$
\frac{\partial T}{\partial r}+B i(T-1)=0, \quad \frac{\partial w}{\partial r}=\frac{\partial \rho}{\partial r}=\frac{\partial C}{\partial r}=0, \quad \Psi=q
$$

$3)$ at the pipe outlet $x=x_{0}=\frac{z_{0}}{r_{0}}-u=B_{\phi}=0, \frac{\partial s}{\partial r}=0, s=\rho ; P s i ; T ; C ; w ; v$,

4) at the pipe inlet $x=0-u=0, \rho=1, B_{\phi}(r, 0)=\frac{1}{r}$ for $r \in[0,1]$ and $w=1, T=C=1, v=0, \Psi=0.5 r^{2}$ for $r \in\left[0, r_{1}\right]$ and $w=0, T=1, C=0$, $\Psi=q, v=4 \frac{\left(r-r_{1}\right)(1-r)}{\left(1-r_{1}\right)^{2}}$ for $r \in\left[r_{1}, 1\right]$; (we have the uniform jet flow for $r<r_{1}$ and and the rotation for $r \geq r_{1}$ with maximal azimuthal velocity 1 when $\left.r=\left(1-r_{1}\right) / 2\right)$.

Here $q=r_{1}^{2} / 2$ is the dimensionless fluid volume, $B i=h r_{0} / \lambda$ is the Biot number, $x_{1}=z_{1} / r_{0}, x_{2}=z_{2} / r_{0}$. Figure 2 represents the sketch of the pilot device (a) and the computational domain (b).
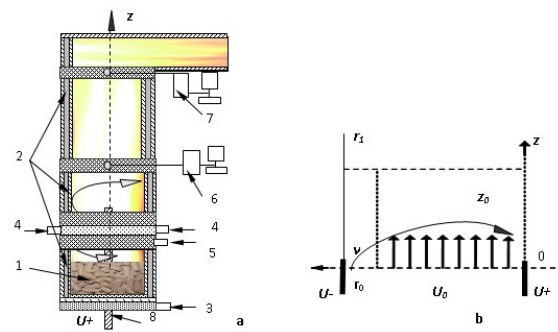

Figure 2. a: the pilot device for experimental studies - 1. biomass gasifier; 2 . water-cooled sections of the combustor; 3. primary axial air supply; 4 . secondary swirling air supply; 5. propane flame supply; 6., 7. orifices for diagnostic tools; 8. positively biased electrode, b: principal computational domain.

\section{The numerical approximations}

We use the uniform grid in space $((M) \times(N+1)):\left\{\left(r_{i}, x_{j}\right), r_{i}=(i-1) h_{r}+h_{r}\right.$, $\left.x_{j}=(j-1) h_{x}\right\}, i=\overline{1, M}, j=\overline{1, N+1}, M h_{r}=1, M_{1} h_{r}=r_{1}, N h_{x}=x_{0}$. 
For time we use the discrete time moments $t_{n}=n \tau, n=0,1, \ldots$. Subscripts $(i, j, n)$ refer to indices $r, x, t$ with mesh spacing and for the approximation of function $u(t, r, x)$ we use the grid function with values $u_{i, j}^{n} \approx u\left(t_{n}, r_{i}, x_{j}\right)$.

For invisced flow we have PDE of hyperbolic type. In this case we use the implicit FDS in time with the upwind differences in space. Here it is an example for $v$-equation of (2.1):

$$
\left(v_{i, j}^{n+1}-v_{i, j}^{n}\right) / \tau+\left(u \delta_{r} v\right)_{i, j}^{n+1}+\left(w \delta_{x} v\right)_{i, j}^{n+1}=0,
$$

where

$$
\begin{aligned}
& \left(u \delta_{r} v\right)_{i, j}=\frac{\left(\left|u_{i, j}\right|+u_{i, j}\right.}{2 h_{r}}\left(v_{i, j}-v_{i-1, j}\right)-\frac{\left(\left|u_{i, j}\right|-u_{i, j}\right.}{2 h_{r}}\left(v_{i+1, j}-v_{i, j}\right), \\
& \left(w \delta_{x} v\right)_{i, j}=\frac{\left(\left|w_{i, j}\right|+w_{i, j}\right.}{2 h_{x}}\left(v_{i, j}-v_{i, j-1}\right)-\frac{\left(\left|w_{i, j}\right|-w_{i, j}\right.}{2 h_{x}}\left(v_{i, j+1}-v_{i, j}\right) .
\end{aligned}
$$

From Taylor expression it follows, that for the upwind approximation

$$
\left(u \delta_{r} v\right)=u \frac{\partial v}{\partial r}-\frac{|u| h_{r}}{2} \frac{\partial^{2} v}{\partial r^{2}}+O\left(h_{r}^{2}\right), \quad\left(w \delta_{x} v\right)=w \frac{\partial v}{\partial x}-\frac{|w| h_{x}}{2} \frac{\partial^{2} v}{\partial x^{2}}+O\left(h_{x}^{2}\right)
$$

and for this approximation we use the artificial viscosities $\frac{|u| h_{r}}{2}, \frac{|w| h_{x}}{2}$. From maximum principle follows that this approximation is stable. The second order derivatives are approximated with central differences. The derivatives at the boundary with the finite differences of first order are approximated.

The reaction-diffusion equations are discretized in the following way:

$$
\begin{aligned}
& \frac{T_{i, j}^{n+1}-T_{i, j}^{n}}{\tau}+\left(u \delta_{r} T\right)_{i, j}^{n+1}+\left(w \delta_{x} T\right)_{i, j}^{n+1}=P_{1}\left(\rho^{-1} \Delta_{h} T\right)_{i, j}^{n+1}+\beta A\left(C \exp \left(-\frac{\delta}{T}\right)\right)_{i, j}^{n}, \\
& \frac{C_{i, j}^{n+1}-C_{i, j}^{n}}{\tau}+\left(u \delta_{r} C\right)_{i, j}^{n+1}+\left(w \delta_{x} C\right)_{i, j}^{n+1}=P_{2}\left(\Delta_{h} C\right)_{i, j}^{n+1}-A\left(C \exp \left(-\frac{\delta}{T}\right)\right)_{i, j}^{n+1},
\end{aligned}
$$

where

$$
\begin{gathered}
\left(\Delta_{h} g\right)_{i, j}=\frac{1}{r_{i} h_{r}^{2}}\left(r_{i+0.5}\left(g_{i+1, j}-g_{i, j}\right)-r_{i-0.5}\left(g_{i, j}-g_{i-1, j}\right)\right) \\
+\frac{1}{h_{x}^{2}}\left(g_{i, j+1}-2 g_{i, j}+g_{i, j-1}\right), \quad g=T, C .
\end{gathered}
$$

For flow with small Mach number we take $\rho_{i, j}=1 / T_{i, j}^{n}$.

\section{The stability for finite difference approximation and numerical method}

For stabilization of the calculations the first equation of (2.1) is approximated by using backward differences formula:

$$
\begin{gathered}
\left(\rho_{i, j}^{n+1}-\rho_{i, j}^{n}\right) / \tau+\left(u \delta_{r} \rho\right)_{i, j}^{n+1}+\left(w \delta_{x} \rho\right)_{i, j}^{n+1}+\rho_{i, j}\left(\left(w_{i, j}-w_{i, j-1}\right) / h_{x}\right. \\
\left.\quad+\left((r u)_{i, j}-(r u)_{i-1, j}\right) /\left(h_{r} r_{i, j}\right)\right)=0
\end{gathered}
$$

but the pressure terms $\frac{\partial p}{\partial r}, \frac{\partial p}{\partial z},(p=\rho T)$ are approximated by the forward differences: $\left((\rho T)_{i+1, j}-(\rho T)_{i, j}\right) / h_{r},\left((\rho T)_{i, j+1}-(\rho T)_{i, j}\right) / h_{x}$. In this case we obtain a fast iteration pocess. 


\subsection{The stability analysis for model equations}

We consider the model equations with constant coefficients for the first three PDEs of (2.1) in the following linear form:

$$
\begin{aligned}
& \frac{\partial \rho}{\partial t}+u_{0} \frac{\partial \rho}{\partial r}+w_{0} \frac{\partial \rho}{\partial x}+\rho_{0} \frac{\partial u}{\partial r}+\rho_{0} \frac{\partial w}{\partial x}=0, \\
& \frac{\partial u}{\partial t}+u_{0} \frac{\partial u}{\partial r}+w_{0} \frac{\partial u}{\partial x}+\frac{T_{0}}{\rho_{0}} \frac{\partial \rho}{\partial r}=0, \\
& \frac{\partial w}{\partial t}+u_{0} \frac{\partial w}{\partial r}+w_{0} \frac{\partial w}{\partial x}+\frac{T_{0}}{\rho_{0}} \frac{\partial \rho}{\partial x}=0,
\end{aligned}
$$

where $u_{0}, w_{0}, \rho_{0} \geq 0, T_{0} \geq 0$ are given constants. Here the periodical boundary conditions in space are used. Similar system of two equations is considered in [19]. We use the following implicit finite difference scheme:

$$
\begin{aligned}
& \frac{\rho_{i, j}^{n+1}-\rho_{i, j}^{n}}{\tau}+\left(u_{0} \delta_{r} \rho\right)_{i, j}^{n+1}+\left(w_{0} \delta_{x} \rho\right)_{i, j}^{n+1}+\rho_{0}\left(\delta_{r} u\right)_{i, j}^{n+1}+\rho_{0}\left(\delta_{x} w\right)_{i, j}^{n+1}=0, \\
& \frac{u_{i, j}^{n+1}-u_{i, j}^{n}}{\tau}+\left(u_{0} \delta_{r} u\right)_{i, j}^{n+1}+\left(w_{0} \delta_{x} u\right)_{i, j}^{n+1}+\frac{T_{0}}{\rho_{0}}\left(\tilde{\delta}_{r} \rho\right)_{i, j}^{n+1}=0, \\
& \frac{w_{i, j}^{n+1}-w_{i, j}^{n}}{\tau}+\left(u_{0} \delta_{r} w\right)_{i, j}^{n+1}+\left(w_{0} \delta_{x} w\right)_{i, j}^{n+1}+\frac{T_{0}}{\rho_{0}}\left(\tilde{\delta}_{x} \rho\right)_{i, j}^{n+1}=0,
\end{aligned}
$$

where $\left(\delta_{r} u\right)_{i, j}=\left(u_{i, j}-u_{i-1 . j}\right) / h_{r},\left(\delta_{x} w\right)_{i, j}=\left(w_{i, j}-w_{i, j-1}\right) / h_{x}$ are the finite backward differences, $\left(\tilde{\delta}_{r} \rho\right)_{i, j}=\left(\rho_{i+1, j}-\rho_{i, j}\right) / h_{r},\left(\tilde{\delta}_{x} \rho\right)_{i, j}=\left(\rho_{i, j+1}-\rho_{i, j}\right) / h_{x}$ are the forward differences. Using the spectral method for the stability investigation of (4.1), the solution is represented as $s_{i, j}^{n}=C_{s} \lambda^{n} \exp \left(\imath\left(k_{r} h_{r}+k_{x} h_{x}\right)\right)$, where $C_{s}$ are unknown constants, $s=(\rho ; u ; w), k_{r}, k_{x}$ are the wave numbers, $\imath$ is the imaginary unit. From (4.1) we get the characteristic equations for obtaining the roots of $\lambda$ in the form of $3 r d$-order matrix-determinant $\operatorname{det} \mathbf{A}=0$, where

$$
\mathbf{A}=\left(\begin{array}{ccc}
G(\lambda) & \rho_{0} \lambda d_{0} & \rho_{0} \lambda d_{0} \\
-\frac{T_{0}}{\rho_{0}} \lambda \bar{d}_{0} & G(\lambda) & 0 \\
-\frac{T_{0}}{\rho_{0}} \lambda \bar{d}_{0} & 0 & G(\lambda)
\end{array}\right)
$$

Here $G(\lambda)=\lambda-1+\lambda d_{0}\left(\left|u_{0}\right|+\left|w_{0}\right|\right), d_{0}=\frac{\tau}{h}(1-\cos (k h)+\imath \sin (k h))$ and $\bar{d}_{0}=\frac{\tau}{h}(1-\cos (k h)-\imath \sin (k h))$ is the complex conjugate expression. For these calculations we have assumed, that $k_{r}=k_{x}=k, h_{x}=h_{r}=h$. The eigenvalues are: $\lambda_{1}=1 /\left(1+d_{0}\left(\left|u_{0}\right|+\left|w_{0}\right|\right)\right)$ with $\left|\lambda_{1}\right|^{2}=1 /\left(1+4 \frac{\tau}{h} \sin ^{2}(k h / 2)\left(\left|u_{0}\right|+\left|w_{0}\right|\right)(1+\right.$ $\left.\frac{\tau}{h}\left(\left|u_{0}\right|+\left|w_{0}\right|\right)\right) \leq 1, \lambda_{2,3}=1 /\left(1+d_{0}\left(\left|u_{0}\right|+\left|w_{0}\right|\right) \pm \imath \sqrt{2 T_{0}}|q|\right)$ with $\left|\lambda_{2,3}\right|^{2}=1 /((1+$ $\left.\left.2 \frac{\tau}{h} \sin ^{2}(k h / 2)\left(\left|u_{0}\right|+\left|w_{0}\right|\right)\right)^{2}+\left(\frac{\tau}{h} \sin (k h)\left(\left|u_{0}\right|+\left|w_{0}\right|\right) \pm \sqrt{2 T_{0}}|q|\right)^{2}\right) \leq 1$, where $q=2 \frac{\tau}{h} \sin (k h / 2)$. Therefore the finite difference scheme (4.1) is unconditionally stable.

If the backward differences are used for the approximation of the derivatives in term $\frac{T_{0}}{\rho_{0}}$, then in the matrix $A$ we have $-\bar{d}_{0}=d_{0}$ and $\lambda_{2,3}=1 /\left(1+d_{0}\left(\left|u_{0}\right|+\right.\right.$ $\left.\left.\left|w_{0}\right|\right) \pm \sqrt{2 T_{0}} d_{0}\right)$. In this case $\left|\lambda_{2,3}\right|^{2}=1 /\left(1+4 b_{0}\left(1+b_{0}^{2}\right) \sin ^{2}(k h / 2)\right)$, where $b_{0}=\frac{\tau}{h}\left(\left|u_{0}\right|+\left|w_{0}\right| \pm \sqrt{2 T_{0}}\right)$. If $\sqrt{2 T_{0}} \leq\left(\left|u_{0}\right|+\left|w_{0}\right|\right)$ the the approximation is unconditionally stable, but for $\sqrt{2 T_{0}}>\left(\left|u_{0}\right|+\left|w_{0}\right|\right)$ (the velocities are smaller 
than the acustic velocity [19]) we have the conditionally stability with the condition $\tau \geq h /\left(\sqrt{2 T_{0}}-\left(\left|u_{0}\right|+\left|w_{0}\right|\right)\right)$ (a non-standard stability condition).

\subsection{The alternating-direction implicit (ADI) method}

For solving the discrete problem

$$
\frac{\boldsymbol{U}^{n+1}-\boldsymbol{U}^{n}}{\tau}=\left(\Lambda_{x}+\Lambda_{r}\right) \boldsymbol{U}^{n+1}+\boldsymbol{f}^{n}, \quad n \geq 0
$$

we use the ADI method of Duglas and Rachford [13] in the form

$$
\begin{aligned}
& \frac{\boldsymbol{U}^{n+0.5}-\boldsymbol{U}^{n}}{\tau}=\Lambda_{x} \boldsymbol{U}^{n+0.5}+\Lambda_{r} \boldsymbol{U}^{n}+\boldsymbol{f}^{n}, \\
& \frac{\boldsymbol{U}^{n+1}-\boldsymbol{U}^{n+0.5}}{\tau}=\Lambda_{r}\left(\boldsymbol{U}^{n+1}-\boldsymbol{U}^{n}\right) .
\end{aligned}
$$

Here the vector $\boldsymbol{U}$ of six components $(\rho, u, w, v, T, C)$ and the scalar function $\Psi$ is used for solving the discrete equations (2.1) and the equation (2.2). $\Lambda_{x}, \Lambda_{r}$ are corresponding differential operators, containing the first and second order derivatives for $\boldsymbol{U}$ with respect to $x$ and $r, \boldsymbol{f}^{n}$ contains all the other functions and derivatives in the PDEs. For solving $\boldsymbol{U}^{n+0.5}$ and $\boldsymbol{U}^{n+1}$ we use the Tomas algorithm in $x$ and $r$ directions respectively. By eliminating the half time step we obtain the previous discrete problem with an approximation error $O\left(\tau^{2}\right)$.

\section{Some numerical results}

In the equations (2.1) the minimal value of the flow density, maximal values of the flow velocity components, the temperature, the reaction rate $R^{*}=$ $A C \exp \left(-\frac{\delta}{T}\right)$, the pressure gradient and the stream function have been calculated.

\subsection{Some results for the full problem}

For the modelling of the full problem, we choose the following parameters: $S=3, x_{0}=2, r_{1}=0.75, r^{*}=h_{y}=0.025, P_{1}=0.1 ; 0.01, P_{2}=0.1 ; 0.01$, $\beta=5, \delta=10, A=50000, B i=0.1, P_{e}=0 ; 0.05 ; 0.1, \tau=0.0008$, It $\leq 18000$ (number of time steps), $N=80, M=40$.

The influence of the molecular diffusivity and thermal conductivity on the main characteristics of the undisturbed flame flow is observed for $P_{e}=0$ and $P_{e}=0.05$. These results show that when the molecular diffusivity $D$ is constant a decrease in thermal conductivity $\lambda\left(P_{1}=0.01, L e=0.1\right)$ leads to an increase in maximal values of the flow velocity components $(w)$, temperature, reaction rates, flow vortices with a decrease in flow density, but for constant thermal conductivity $\lambda$ the decrease in molecular diffusivity $D\left(P_{2}=0.01, L e=10\right)$ results in an increase of maximum density and in a decrease of velocity, pressure gradient, temperature, reaction rate and flow vortices.

The results of the numerical simulation show that the electric field influence on the main flame characteristics is determined by the length of the axially 
inserted electrode (see Figure 3). For $x_{1}=x_{2}=1, P_{1}=P_{2}=0.01, P_{e}=0$ we have obtained Figure 3 (a) and the following numerical results $(I t=1723)$ : $p \in[0.153,4.40], w=\in[0,5.797], u \in[0,2.786], \rho \in[0.0305,1], T \in[1,5.994]$, $R_{\max }^{*}=298.76, \Psi_{\max }=0.2924$ (a small vortex), $T_{a}=5.1927\left(T_{a}\right.$ is the averaged value of temperature, $q=0.2812)$.

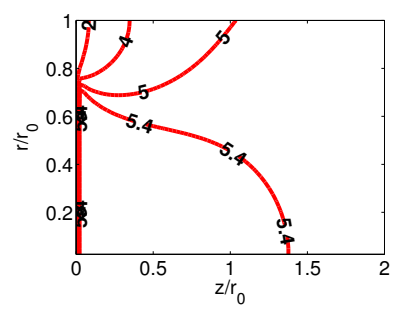

(a) for $P_{e}=0$

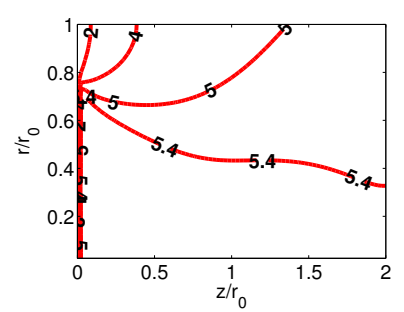

(b) for $P_{e}=0.05$

Figure 3. Temperature levels.

If $P_{e}=0.05$ (see Figure $3(\mathrm{~b})$ ) then $p \in[0.162,4.32], w \in[0,11.21]$, $u \in[-0.25,2.718], \rho \in[0.0321,1], T \in[1,5.995], R_{\max }^{*}=298.92, \Psi_{\max }=$ $0.2924, T_{a}=5.1986$.

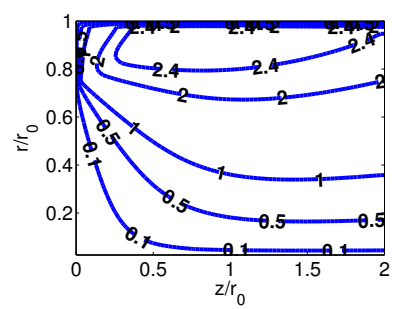

(a) for $P_{e}=0$

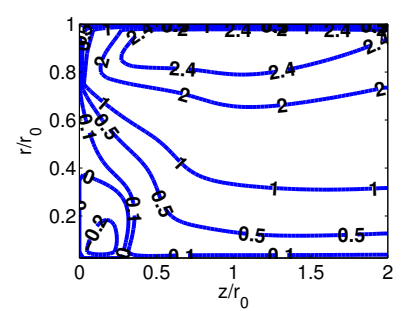

(b) for $P_{e}=0.05$

Figure 4. Levels of radial velocity.

In Figure 4 we can see that the electric field in the flame reaction zone $\left(P_{e}=0.05\right)$ disturbs the stream lines, initiates the formation of small vortices and the radial velocity changes the sign.

In Figure 5, 6 we represent the levels of the electric current $\left(x_{1}=0.25, x_{2}=\right.$ 2 ) and maximal temperature time dependence for $\beta=0.5, P_{1}=0.01, P_{2}=0.1$; the flame temperature $(\mathrm{t}=0.2 \mathrm{~s})$ rapidly increases to its maximum value.

\subsection{Some numerical experiments with the reaction-diffusion equa- tion}

For the fixed values of velocity $u=v=0, w=1$ and $P_{1}=P_{2}=0.1, r_{1}=0.5$, the heat-reaction problem is solved numerically in two ways: $\rho=1 / T$ (small 


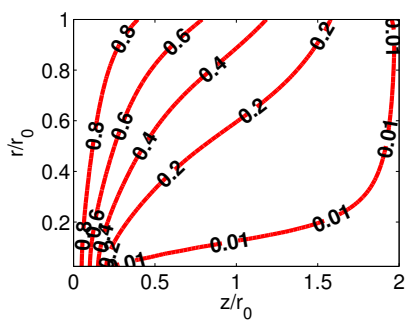

Figure 5. Levels of electric current for $x_{1}=0.25, x_{2}=2$.

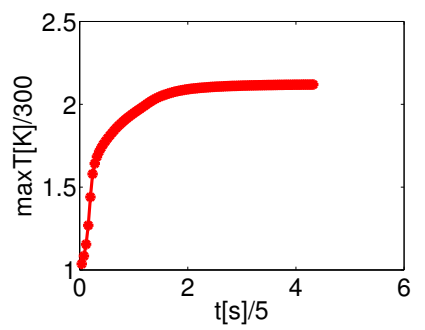

Figure 6. Maximal temperature depending on time $\mathrm{t}$ by $\beta=0.5, P_{1}=0.01, P_{2}=0.1$.

Mach numbers for compressible fluid) and $\rho=1$ (incompressible flow). If $\rho=$ $1 / T$, we have the maximal and averaged values of temperature: $T_{\max }=2.957$, $T_{a}=1.827$ and the value of $R_{\max }^{*}=252.20$. For $\rho=1$ we have $T_{\max }=5.969$, $T_{a}=3.319, R_{\max }^{*}=593.31$. The calculation results are presented in Figure 7 . For $r_{1}=0.75$ we have the corresponding $T_{\max }=6.806, T_{a}=5.168, R_{\max }^{*}=$ $593.51(\rho=1), T_{\max }=3.320, T_{a}=2.890, R_{\max }^{*}=255.84(\rho=1 / T)$.

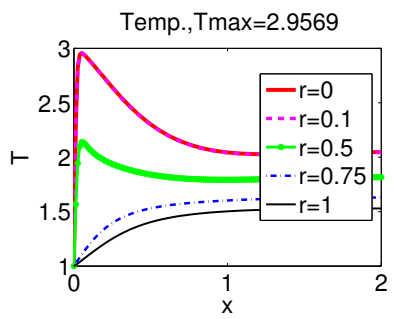

(a) for $\rho=1 / T$

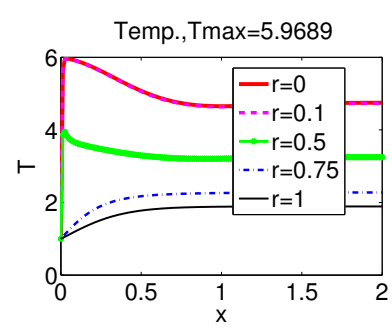

(b) for $\rho=1$

Figure 7. Profiles of temperature depending on $x$.

\subsection{Solving 1-D heat-reaction problem with MATLAB solver "pdepe"}

Using the two simple reactions from (2.1) we obtain following $1 \mathrm{D}$ reactiondiffusion problem for temperature $T(x, t)$ and 2 reaction concentrations $C_{1}(x, t), C_{2}(x, t)(\rho=1, u=0)$ :

$$
\begin{aligned}
& \frac{\partial T}{\partial t}+w \frac{\partial T}{\partial x}=P_{1} \frac{\partial^{2} T}{\partial x^{2}}+\beta_{1} A_{1} C_{1} \exp \left(-\frac{\delta_{1}}{T}\right)+\beta_{2} A_{2} C_{2} \exp \left(-\frac{\delta_{2}}{T}\right), \\
& \frac{\partial C_{1}}{\partial t}+w \frac{\partial C_{1}}{\partial x}=P_{2} \frac{\partial^{2} C_{1}}{\partial x^{2}}-A_{1} C_{1} \exp \left(-\frac{\delta_{1}}{T}\right), \\
& \frac{\partial C_{2}}{\partial t}+w \frac{\partial C_{2}}{\partial x}=P_{2} \frac{\partial^{2} C_{2}}{\partial x^{2}}-A_{2} C_{2} \exp \left(-\frac{\delta_{2}}{T}\right), \\
& t \in\left(0, t_{f}\right), x \in(0, L), T(0, t)=1, C_{1}(0, t)=0.8, C_{2}(0, t)=0.2, \\
& \frac{\partial(s(L, t)}{\partial x}=0, s=T, C_{1}, C_{2}, T(x, 0)=1, C_{1}(x, 0)=C_{2}(x, 0)=\exp (-\alpha x),
\end{aligned}
$$


where $A_{1}=A=5.10^{4}, A_{2}=5.10^{5}, \beta_{1}=\beta=5, \beta_{2}=1, \delta_{1}=\delta=10$, $\delta_{2}=15, t_{f}=1, w=0,1,2,3,4, P_{1}=0.1,0.01, P_{2}=0.1,0.01,0.001, \alpha=$ $0,1,2,3,4,5,6, L=2,4, \alpha \in[0,6]$ is the parameter for the initial fuel amount in the combustion.

The results of calculation using 2 reactions $\left(L=2, \alpha=6, P_{1}=P_{2}=0.1\right.$ with $T_{\max }=5.269, w=1(T(2,1)=1.248)$ and $\left.w=4(T(2,1)=5.200)\right)$ are represented in the Figure 8.

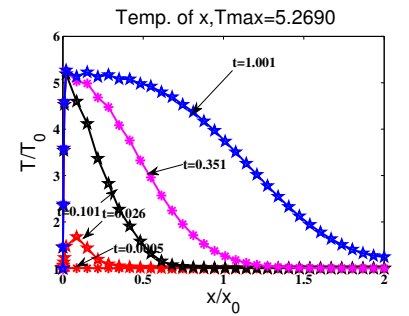

(a) for $w=1, \alpha=6, P_{1}=P_{2}=$ 0.1

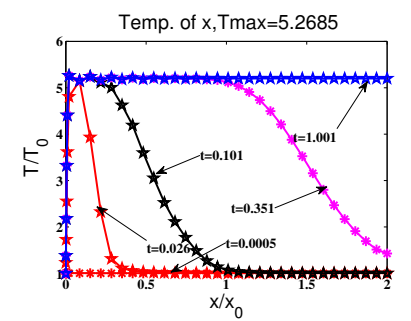

(b) for $w=4, \alpha=6, P_{1}=P_{2}=$ 0.1

Figure 8. Profiles of temperature depending on $x$ in fixed time $t$.

Using one reaction $\left(C_{2}(0, t)=0, C_{1}(0, t)=1\right)$ : for $w=1, T_{\max }=6.0881$, $T(2,1)=1.295$ and for $w=4, T_{\max }=6.114, T(2,1)=1.248$. In Figures 9, 10 (one reaction) we can see the surface in $(x, t)$ plane at $w=4, L=4, \alpha=$ $6 P_{1}=P_{2}=0.1,\left(T_{\max }=6.281, T(4,1)=4.085\right)$ and profile of temperature for $w=3, L=2, P_{1}=0.01, P_{2}=0.001\left(T_{\max }=5.690, T(2,1)=4.000\right)$.

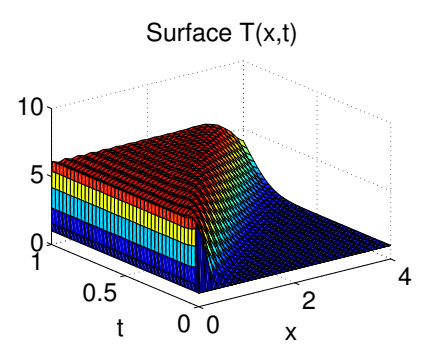

Figure 9. Temperature depending on $(x, t)$ for $w=4, L=$ $4, \alpha=6, P_{1}=P_{2}=0.1$.

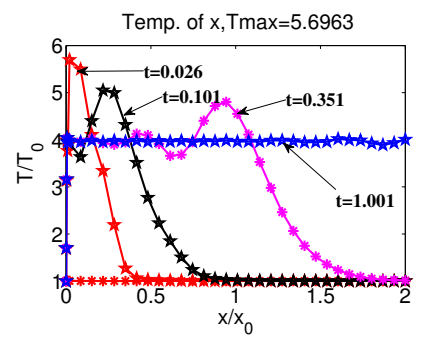

Figure 10. Profile of temperature depending on $x$ in fixed time $t$ for $w=3, L=2, \alpha=$ $6, P_{1}=0.01, P_{2}=0.001$.

\subsection{Some results obtained with MATLAB solver "pdepe"}

For $P_{1}=P_{2}=0.1, L=2$ we obtain the following results for the temperature $T\left(2, t_{f}\right)$ in outlet $(x=2)$ (see Table 1$)$ :

1) for constant initial conditions for $\mathrm{C}$ with $\left(\alpha=6, t_{f}=1\right)$ an increase in velocity $w$ leads to an increase in the value of temperature $T(2,1)$, 
2 ) for constant velocity $\left(w=1, t_{f}=1\right)$ a decrease in parameter $\alpha$ (the initial fuel amount in combustion is increased) leads also to an increase in the value of temperature $T(2,1)$, item for constant $\alpha=6, w=0$ an increase in the time segment $t_{f}$ leads to an increase in temperature $T\left(2, t_{f}\right)$.

Table 1. The values $T\left(2, t_{f}\right)$ depends on $w, \alpha, t_{f}$

\begin{tabular}{rrrrrrrr}
\hline \multicolumn{2}{c}{$t_{f}=1, \alpha=6$} & & \multicolumn{2}{c}{$w=t_{f}=1$} & & \multicolumn{2}{c}{$w=0, \alpha=6$} \\
\cline { 1 - 2 } \cline { 6 - 7 }$w$ & $\mathrm{~T}(2,1)$ & & $\alpha$ & $\mathrm{T}(2,1)$ & & $t_{f}$ & $T\left(2, t_{f}\right)$ \\
\hline 0 & 1.002 & & 6 & 1.295 & & 1 & 1.002 \\
1 & 1.295 & & 3 & 1.694 & & 10 & 2.630 \\
2 & 4.396 & & 1 & 3.204 & & 20 & 4.177 \\
3.5 & 6.000 & & 0 & 6.000 & & 100 & 6.000 \\
\hline
\end{tabular}

The maximal temperature depends on molecular diffusivity and thermal conductivity $\left(P_{1}, P_{2}\right)$, an example for $L=2, t_{f}=1, \alpha=6, w=1: P_{1}=$ $P_{2}=0.1, T_{\max }=6.09, T(2,1)=1.295, P_{1}=P_{2}=0.01, T_{\max }=6.27$, $T(2,1)=1.000, P_{1}=0.1, P_{2}=0.01, T_{\max }=4.46, T(2,1)=1.196, P_{1}=0.01$, $P_{2}=0.001, T_{\max }=5.69, T(2,1)=1.020$.

\subsection{Solving 1-D stationary reaction-diffusion problem with MAT- LAB solver "bvp4c"}

For stationary reaction-diffusion equation $5.1\left(A_{1}=5.10^{4}, A_{2}=5.10^{8}, \delta_{1}=10\right.$, $\left.\delta_{2}=20, \rho=1, w=0, T=T(x), C_{1}=C_{1}(x), C_{2}=C_{2}(x), x \in[0,2]\right)$ with BCs $T(0)=1, C_{1}(0)=0.8, C_{2}(0)=0.2, T(2)^{\prime}=C_{1}^{\prime}(2)=C_{2}^{\prime}(2)=0$, by multiplying both second equations by $\beta_{1}, \beta_{2}$ and summing them, we get the equations

$$
\begin{aligned}
& L e T^{\prime \prime}(x)+\beta_{1} C_{1}^{\prime \prime}(x)+\beta_{2} C_{2}^{\prime \prime}(x)=0, \\
& \operatorname{Le}(T(x)-1)+\beta_{1}\left(C_{1}(x)-0.8\right)+\beta_{2}\left(C_{2}(x)-0.2\right)=0, \\
& \operatorname{LeT}^{\prime}(x)=-\beta_{1} C_{1}^{\prime}(x)-\beta_{2} C_{2}^{\prime}(x) .
\end{aligned}
$$

In the limit case $x \rightarrow 2$ it follows that the maximal temperature is $T_{\max }=$ $T(2)=1+\frac{\beta_{1} 0.8+\beta_{2} 0.2}{L e}, L e=\frac{P_{1}}{P_{2}}$. We have obtained in Matlab, that the increase in the axial velocity $w$ leads to an increase for $L e>1$ and the desrease for $L e<1$ in $T_{\max }$. For $L e=1, T_{\max }$ does not depend on $w$; increase in $w$ leads to the descrease in thickness of the boundary layers $T^{\prime}(0)$ and $C^{\prime}(0)$ (see Table 2 for one reaction and $\beta=1$ ).

In the Figure 11 the profile of temperature and concentration for one reaction we can see depending on $x$ for $w=0.5, P_{1}=0.005, P_{2}=0.01$ and $\beta=0.1 ; 0.2 ; 0.3 ; 0.4$.

\section{Conclusions}

- The stability for discreet problem of the model equations has been investigated. 
Table 2. The values of $T_{\max }, T^{\prime}(0), C^{\prime}(0)$ depend on $P_{1}, P_{2}, w$

\begin{tabular}{rrrrrr}
\hline$P_{1}$ & $P_{2}$ & $\mathrm{w}$ & $T_{\max }$ & $\mathrm{T}^{\prime}(0)$ & $\mathrm{C}^{\prime}(0)$ \\
\hline 0.01 & 0.01 & 0.0 & 2.00 & 68.6 & -68.6 \\
0.01 & 0.01 & 1.0 & 2.00 & 9.15 & -9.15 \\
0.02 & 0.01 & 0.0 & 1.50 & 17.6 & -35.3 \\
0.02 & 0.01 & 1.0 & 1.83 & 12.9 & -9.02 \\
.005 & 0.01 & 0.0 & 3.00 & 98.1 & -78.3 \\
.005 & 0.01 & 0.5 & 2.54 & 84.9 & -69.5 \\
\hline
\end{tabular}

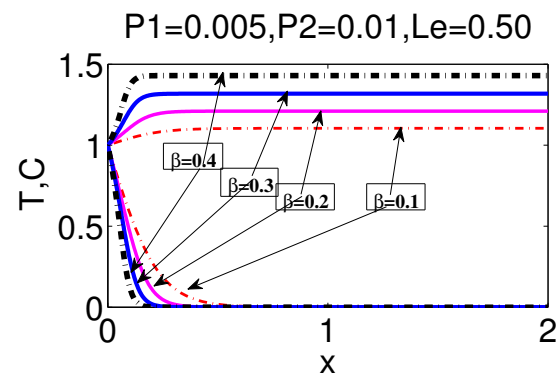

Figure 11. Profile of temperature and concentration depending on $x$ for $\beta=0.1 ; 0.2 ; 0.3 ; 0.4$.

- For the diffusion-reaction problem, the influences of maximal temperature in pipe and temperature at outlet are obtained.

- The maximal temperature depends on molecular diffusivity and thermal conductivity.

- The maximal axial velocity depends on Lewis number value.

- Increase in electrodynamical force parameter $P_{e}$ leads to an increase in maximal velocity and in temperature.

\section{Acknowledgement}

This work was partially supported by the grant 623/2014 of the Latvian Council of Science and ERAF project Nr.1.1.1.1/16/A/004.

\section{References}

[1] A. Aboltins, H. Kalis, K. Pulkis, J. Skujans and I. Kangro. Mathematical modelling of heat transfer problem for two layered gypsum board products exposed to fire. In Latvia J. Palabinskis, Latvia University of Agriculture(Ed.), Proc. of of the 16-th Int. Conf. on Engineering for Rural Development (ERDev17), Jelgava, Latvia, May. 24-26, 2017, pp. 1369-1376, Latvia University of Agriculture, Faculty of Engineering, 2017. https://doi.org/10.22616/ERDev2017.16.N312. 
[2] M. Abricka, I. Barmina, R. Valdmanis, M. Zake and H. Kalis. Experimental and numerical studies on integrated gasification and combustion of biomass. Chemical Engineering Transactions, 50:127-132, 2016.

[3] N. Ahmed and K.Kr. Das. Effects of heat absorption and chemical reaction on a three dimensional MHD convective flow past a porous plate. Int. J.of Phys. Sciences, 8(39):1907-1922, 2013.

[4] I. Barmina, A. Kolmickovs, R. Valdmanis, M. Zake and H. Kalis. Experimental and numerical studies of electric field effects on biomass thermo-chemical conversion. Chemical Engineering Transactions, 50:121-126, 2016.

[5] I. Barmina, A. Komickova, R. Valdmanis and M. Zake. Combustion dynamics of swirling flame at thermo chemical conversion of biomass. Chemical Engineering Transaction, 43:649-654, 2015.

[6] I. Barmina, R. Valdmanis, H. Kalis and M. Marinaki. Experimental and numerical study of the development of swirling flow and flame dynamics and combustion characteristics at biomass thermo-chemical conversion. In Latvia J. Palabinskis, Latvia University of Agriculture(Ed.), Proc. of of the 16-th Int. Conf. on Engineering for Rural Development (ERDev17), Jelgava, Latvia, May. 24-26, 2017, pp. 68-74, Latvia University of Agriculture, Faculty of Engineering, 2017. https://doi.org/10.22616/ERDev2017.16.N013.

[7] I. Barmina, R. Valdmanis, M. Zake, H. Kalis, M. Marinaki and U. Strautins. Magnetic field control of the combustion dynamics. Latvian Jour. of Physics and Technical Sciences, 53(4):36-47, 2016. https://doi.org/10.1515/lpts-20160027.

[8] I. Barmina, M. Zake, H. Kalis and M. Marinaki. Electic field effects on gasification / combustion at thermo-chemical conversion of biomass mixtures. In Latvia J. Palabinskis, Latvia University of Agriculture(Ed.), Proc. of of the 16-th Int. Conf. on Engineering for Rural Development (ERDev17), Jelgava, Latvia, May. 24-26, 2017, pp. 60-67, Latvia University of Agriculture, Faculty of Engineering, 2017. https://doi.org/10.22616/ERDev2017.16.N012.

[9] F. Battaglija, K. McGrattan, R.G. Rehm and H.R. Baum. Simuling fire whirls. Combustion Theory and Modelling, 4(2):123-138, 2000. https://doi.org/10.1088/1364-7830/4/2/303.

[10] V. Bayona and M. Kindelan. Propagation of premixed laminar flames in 3D narrow open ducts using PBF- generated finitedifferences. Combustion Theory and Modelling, 17(5):789-803, 2013. https://doi.org/10.1080/13647830.2013.801519.

[11] V.V. Boyarevitch, Y.Zh. Freiberg, E.I. Shilova and E.V. Therbinyn. Electrovortexed flows. Zinatne Press,Riga, 1985, in Russian.

[12] J.J. Choi, Z. Rusak and A.K. Kapila. Numerical simulation of premixed chemical reactions with swirl. Combustion theorie and modelling, 6(11):863-887, 2007.

[13] J. Douglas and R. Rachford. On the numerical sulution of heat conduction problems in two and three space variables. Trans. Amer.Math.Soc., 82(2):421439, 1956. https://doi.org/10.1090/S0002-9947-1956-0084194-4.

[14] A.K. Gupta, D.G. Lilley and N. Syred. Swirl Flows. Abacus Press, 1984.

[15] H. Kalis, I. Barmina, M. Zake and A. Koliskins. Mathematical modelling and experimental study of electrodynamic control of swirling flame flows. In Latvia J. Palabinskis, Latvia University of Agriculture(Ed.), Proc. of of the 15-th Int. 
Conf. on Engineering for Rural Development (ERDev16), Jelgava, Latvia, May. 25-27, 2016, pp. 134-141, Latvia University of Agriculture, Faculty of Engineering, 2016.

[16] H. Kalis and M. Marinaki. Numerical study of 2D MHD convection around periodically placed cylinders. Int. Jour. of Pure and Applied Mathematics, 110(3):503-517, 2016. https://doi.org/10.12732/ijpam.v110i3.10.

[17] H. Kalis, M. Marinaki, U. Strautins and Lietuvietis. On the numerical simulation of the combustion process with simple chemical reaction. In Dmitri Meshumayev and Bength Sunden(Eds.), Proc. of the 7-th Baltic Heat Transfer Conference, Tallinn, Estonia, Aug. 24-26, 2015, Baltic Heat Transfer Conference BHTC, pp. 175-180. Tallinn University of Technology, 2015. ISBN 978-9949-23-817-0.

[18] H. Kalis, M. Marinaki, U. Strautins and Lietuvietis. On the numerical simulation of the vortex breakdown in the combustion process with simple chemical reaction and axial magnetic field. Int. Jour. of Differential Equations and Applications, 14(3):235-250, 2015.

[19] V.M. Kovenya and N.N. Yanenko. The method of decomposition in gaz dynamical problems. Nauka Press, Novosibirsk, 1981, in Russian.

[20] D.G. Lilley. Swirl flows in combustion: A review. AIAA Journal, 15(8):10631078, 1977. https://doi.org/10.2514/3.60756.

[21] D. Meeker. Finite Element Method Magnetics. Version 4.2, User's Manual, 2015.

[22] V. Mittal, H. Pitsh and F. Egolfopoulos. Assessment of counterflow to measure laminar burning velocities using direct numerical simulation. Combustion Theory and Modelling, 16(3):419-433, 2012. https://doi.org/10.1080/13647830.2011.631033.

[23] N. Syred and J.M. Beer. Combustion in swirling flows: A review. Combustion and Flame, 23(2):143-201, 1974. https://doi.org/10.1016/0010-2180(74)900571.

[24] A.B. Watathin, G.A. Lyubimov and S.A. Regirer. Magnetohydrodynamical flows in the canalls. Nauka Press, Moscou, 1970, in Russian.

[25] A. Zlotnik and R. Čiegis. A converse stability condition is necessary for a compact higher order scheme on non-uniform meshes. Applied Mathematics Letters, 80:35-40, 2018. https://doi.org/10.1016/j.aml.2018.01.005.

[26] A. Zlotnik and V. Gavrilin. On quasi-gasdynamic system of equations with general equations of state and its application. Mathematical Modelling ana Analysis, 16(4):509-526, 2011. https://doi.org/10.3846/13926292.2011.627382. 